\section{DSL-002 A PHARMACOECONOMIC COMPARISON BETWEEN A COUNTY HOSPITAL IN CHANIA AND A CENTRAL HOSPITAL IN ATHENS, GREECE}

doi:10.1136/ejhpharm-2013-000276.245

'D Makridaki, 'C Allagianni, 'R Skountzou, ${ }^{2} \mathrm{M}$ Petrogonas, ${ }^{2} \mathrm{E}$ Rinaki, ${ }^{2} \mathrm{~L}$ Tzimis. 'Sismanoglio GH, Pharmacy, Athens, Greece; ${ }^{2}$ Chania GH, Pharmacy, Chania, Greece

Background 'Agios Georgios' Chania General Hospital (CGH) on the island of Crete has 460 beds and Sismanoglio General Hospital (SGH), in the capital of Greece, Athens, has 439 beds. In the Greek National Health System the uninsured poor patients receive their dugs free of charge from the hospital pharmacies.

Purpose To compare the pharmacoeconomic profiles of the two hospitals.

Materials and Methods We examined the pharmacoeconomic data for the first half of 2011. Data were extracted from the Hospital Information Systems.

Results 14,998 patients were hospitalised in CGH and 15,520 patients in SGH with a mean number of nursing days 3.99 vs. 3.55 .

The total cost of drugs was $€ 6,705,297$ vs. $€ 4,933,028$ ( $P<0.05$ ) respectively.

The drugs cost for the inpatients was $€ 5,034,701$ vs. $€ 3,965,127$ and the mean cost per impatient per nursing day was $€ 77.67$ vs. $€ 67.23$.

The drugs cost for the insured outpatients was $€ 1,452,668$ vs $€ 713,203$ (1,595 prescriptions vs. 1,152, $\mathrm{P}<0.05$ ), and the mean cost per prescription was $€ 909.42$ vs. $€ 619.10$ ( $\mathrm{P}<0.05$ ).

For the uninsured outpatients the drugs bill was $€ 217,928$ vs. $€ 254,694$ (3,506 prescriptions vs. $2,016 \mathrm{P}<0.05)$ and the mean prescription cost was $€ 62.16$ vs. $€ 126.34$ ( $\mathrm{P}<0.05$ ).

The percentage cost for the main categories of drugs were: cytostatics $16.50 \%$ vs. $10.65 \%$, antibiotics $21.65 \%$ vs. $24.51 \%$, antirheumatics $7.54 \%$ vs. $4.55 \%$, cardiovascular $5.57 \%$ vs. $3.98 \%$ and erythropoietins $11.45 \%$ vs. $3.11 \%$ ( $\mathrm{P}<0.05)$.

The ratio of generics to patented medicines was $40.32 \%: 59.68 \%$ and $39.14 \%: 60.86 \%$

Conclusions We found statistical differences among the pharmacoeconomic data of the two hospitals. In SGH, HIV+ patients are served $(27.47 \%$ of uninsured and $47.35 \%$ of insured outpatients) and this is reflected in the increased cost of the outpatients while erythropoietins and cytostatics cost differences are related to the hospital departments (Oncology, Haematology, Pulmonary clinics), the different DRGs and treatment protocols followed in each hospital.

No conflict of interest.

\section{DSL-003 AUTOMATION OF DRUG DISTRIBUTION: IMPACT ON ERROR RATE AND DISTRIBUTION SPEED}

\section{doi:10.1136/ejhpharm-2013-000276.246}

O François, L Carrez, L Gschwind, L Cingria, N Vernaz-Hegi, P Bonnabry. University Hospitals of Geneva, Pharmacy, Geneva, Switzerland

Background Human reliability is limited and information technology has the potential to improve the safety of the medication process. In July 2011, a robot (ROWA/ARX) was implemented in our hospital pharmacy to reduce error rates and improve the efficiency of our global drug distribution.

Purpose To evaluate the impact of this automation on distribution errors and workload efficiency.

Materials and Methods Approximately $52 \%$ of the dispensary stock (1126 articles, 50,000 boxes) is managed by the robot.

1. Distribution errors: content accuracy of random orders was verified before and after the implementation of the robot. Errors were classified in three categories: wrong drug, missing drug/quantity or additional quantity.

2. Workload efficiency: time to prepare a sequence of orders manually or with the robot was measured.

\section{Results}

1. Manual dispensing error rate was $0.93 \%$ ( $n=5805$ ordered lines; wrong drug: $0.36 \%$, missing drug/quantity: $0.31 \%$, additional quantity: $0.26 \%)$. By decreasing this error rate to $0.27 \%$ ( $n=5840$; only conveyor errors leading to missing drug/quantity and additional quantity), the automation avoided more than 4500 errors each year.

2. With the distribution of 880 boxes of drugs/hour (reduced to 630 when the automated 'Pro-log' filling system was working simultaneously), the robot significantly increased the distribution speed in comparison with the manual picking (303 boxes/hour).

Conclusions This reorganisation contributed to safer and more efficient distribution of drugs. No more incorrect picking of medicines occurred thanks to the high reliability of the robot. Remaining errors could still be reduced by improving the conveyor software. With one single person operating the robot, 2 full-time equivalents were saved, leading to an estimated return on investment in 4.5 years. For medicines remaining outside the robot (i.e. controlled drugs, cold chain drugs or those with an unusual size, shape or weight), a scanning system will be introduced and evaluated by the same protocol.

No conflict of interest.

\section{DSL-004 AUTOMATION OF STORAGE AND DISPENSING: WHAT SYSTEM SHOULD WE IMPLEMENT?}

doi:10.1136/ejhpharm-2013-000276.247

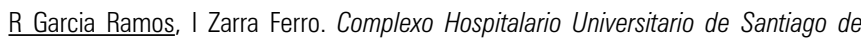
Compostela, Pharmacy, Santiago de Compostela, Spain

Background Innovation and new technologies help reduce the rate of medication errors and maximise efficiency in the drug administration system thus improving the safety and quality of patient care. In the market there are various automation systems, all of which are costly.

Purpose To analyse two storage and dispensing automation systems in order to make a decision to improve the safety, efficiency and quality of medicines use in our hospital.

Materials and Methods Review of two systems: A) fully integrated robotic automation (fully enclosed storage modules that automatically generate individual dosage units (DUs) grouped into rings per patient), and B) system with different components (semiautomatic storage and cart-filling system, plus storage tanks filling, automatic dispensing systems (DAS) in inpatient units, plus outpatient medicines automation and repackaging). We analysed the resources currently available and the benefits of the two systems. DUs consumed in 2011 were examined and classified by pharmaceutical form, volume, storage conditions and whether they can be dispensed to outpatients or not. High volume solutions and enteral nutrition were excluded. The costs used in the analysis are the sum of the quotes received from suppliers, excluding maintenance costs. The same level of human resources was assumed. Costs were expressed as additional costs per number of DUs dispensed under each system.

Results 16.213.352 DUs were dispensed in 2011 in connexion with 2971 drugs (40\% could be dispensed to outpatients). Advantages and disadvantages of the two systems are listed in the Table.

Conclusions The integrated robotics system (system A) appears to be a safer, more versatile and more efficient system providing more information than system $B$, which provides greater accessibility for nursing. The cost analysis is slightly favours system A. One 
limitation of the study is that the costs of maintenance and the human resources reengineering required need to be further explored.

Abstract DSL-004 Table 1

\begin{tabular}{|c|c|c|}
\hline & \multicolumn{2}{|c|}{ Advantages and disadvantages of the two systems } \\
\hline & System A & System B \\
\hline \multirow[t]{3}{*}{ SAFETY } & $\begin{array}{l}\text { All DUs can be unequivocally identified } \\
\text { with batch expiry date }\end{array}$ & $\begin{array}{l}\text { Partial identification with batch barcode } \\
\text { and expiry date }\end{array}$ \\
\hline & $\begin{array}{l}\text { Complete record, including batch, } \\
\text { administration by scanner }\end{array}$ & $\begin{array}{l}\text { Record drug administration with bar } \\
\text { code without batch }\end{array}$ \\
\hline & Closed system & Partially open systems, error risks \\
\hline \multirow[t]{4}{*}{ EFFICIENCY } & $\begin{array}{l}\text { Entire integrated system including } \\
\text { outpatients and elderly residences }\end{array}$ & $\begin{array}{l}\text { Immediate availability of nursery doses } \\
\text { needed to the patient }\end{array}$ \\
\hline & Full return of unmanaged DUs & \\
\hline & $\begin{array}{l}\text { Allows automatic checking of expiry } \\
\text { dates }\end{array}$ & Full expiry date control is difficult \\
\hline & High cost & High cost \\
\hline QUALITY & $\begin{array}{l}\text { Complete record of all movements of } \\
\text { both drugs and users }\end{array}$ & $\begin{array}{l}\text { Partial recording of users, batches, } \\
\text { drugs in drug use chain }\end{array}$ \\
\hline $\begin{array}{l}\text { Additional cost } \\
\text { per DU (euros) }\end{array}$ & 0.19 & 0.20 \\
\hline
\end{tabular}

No conflict of interest.

\section{DSL-005 COMPARATIVE STUDY OF THE COST OF ERYTHROPOIETIC FACTORS, ORIGINAL MEDICINES AND BIOSIMILARS IN FRENCH CARE FACILITIES}

doi:10.1136/ejhpharm-2013-000276.248

'D Karouby, 'C Vallet, ${ }^{2} \mathrm{~F}$ Bocquet, ${ }^{3 P}$ Paubel. ' 'GROUPE HOSPITALIER PARIS SAINT JOSEPH, Pharmacy, Paris, France; '2Agence Générale des Produits de Santé, Pharmacy, Paris, France, ${ }^{3}$ Université Paris-Descartes, Pharmacy, Paris, France

Background The patent expiries of leading biological products and the development of biosimilars create opportunities for cost savings. No studies have been carried out in the French hospital market.

Purpose To perform a cost saving modelling analysis and investigate the potential factors that could affect the price of drugs.

Materials and Methods We carried out a comparative study in French healthcare facilities, representing about $65 \%$ of national hospital beds, of the price of erythropoietic factors. The data were collected on procurement procedures operative as of 1 January 2012.

Results 25 care facilities agreed to participate in the study. The overall sales turnover reached $€ 15 \mathrm{M}$. Biosimilars represent less than $1 \%$ market share. All the establishments granted a discount of between $5 \%$ and $69 \%$ on the prices fixed by negotiation between the Comité Economique des Produits de Santé and the manufacturers, depending on the category (drugs, biosimilars or original biopharmaceuticals). The average discounts ranged from $11 \%$ to $73 \%$. Binocrit, the main biosimilar represented was $25.6 \%$ less expensive than its original medicine Eprex. Based on French hospital financing, we show a $24.7 \%$ cost saving if a high interchangeability rate is adopted. Some participants could save up to $50 \%$ of their budget.

We identified and analysed three criteria known to have a farreaching effect on the drugs price. We observe no or little effect of the type of procurement procedure and specified quantity of medicine. The starting date of the contract is the primary criterion when purchasing drugs. The impact of these criteria varied depending on the drug in question and no general conclusions about medicines could be drawn.

Conclusions The market for biosimilars is growing at a faster rate than the global prescription-drug market. Many top-selling biologicals are due to lose patent protection over the next few years. The great potential for cost savings apparent in our study could be investigated in other countries.

No conflict of interest.

\section{DSL-006 COST ANALYSIS OF ADULT PARENTERAL NUTRITION SYSTEMS: THREE-COMPARTMENT BAG VERSUS CUSTOMISED}

doi:10.1136/ejhpharm-2013-000276.249

'D Berlana, 'P Sabin, ${ }^{2} \mathrm{~J}$ Rius, ${ }^{2} \mathrm{E}$ Llop, ${ }^{3} \mathrm{R}$ Romero, 'E Marquez, ${ }^{2} \mathrm{JA}$ Schoenenberger, 1J Martinez. 'Hospital Univ. Vall Hebron, Pharmacy, Barcelona, Spain; ${ }^{2}$ Hospital Arnau Vilanova, Pharmacy, Lleida, Spain; ${ }^{3}$ Hospital Gregorio Marañon, Pharmacy, Madrid, Spain

Background Parenteral nutrition (PN) is a costly technology used widely to provide nutrition to patients who have an inaccessible or non-functioning intestine. Two all-in-one systems currently being used are customised formulations, prepared by hospital pharmacies, and three-compartment bags.

Purpose To provide a systematic cost comparison of the two all-inone PN systems: individualised (made from nutrient solutions) versus manufactured (made from three-compartment bag), both prepared in hospital pharmacies.

Materials and Methods We conducted a prospective study to analyse the total cost of PN bags, accounting for all of the processes involved in preparing and delivering them (the cost of manpower, nutrition solutions, medical supplies and quality controls) in three different healthcare settings. To compare therapeutic alternatives of equivalent nutritional value, the study was performed for the most frequently-employed formulation, which was similar to commercial preparations. A univariate sensitivity analysis was performed to evaluate the impact of different rates of use of three-compartment PN bags.

Results 157 routine acts of PN bag preparation (65 hospital compounded and 92 three-compartment) were observed and timed over 9 days. Total costs of the 157 PN bags were included in the study. Mean costs of hospital-compounded bags were higher than threecompartment bags, $51.16 \pm 5.63 €$ versus $39.69 \pm 3.00 €$ respectively $(p<0.01)$. Manpower costs were responsible for the majority of the differences found (70\%). In scenarios using a three-compartment system for $30 \%, 70 \%$ and $90 \%$ of $\mathrm{PN}$ provision, a cost savings of $4.3 \%, 10.1 \%$ and $12.9 \%$ respectively could be achieved. Greatest rates of changing from hospital compounded bags (70\% and $90 \%)$, in a hospital with 1,800 PN bags/year, might reduce the annual budget by $9306 €$ and $11,964.8 €$, respectively. Meanwhile, in a large facility the savings for 8,000 TPN days would be $64,248 €$ and $82,605 €$, respectively.

Conclusions Since we need to reduce the costs of effective treatments, three-compartment bags could be used for standard adult PN to save money.

No conflict of interest.

\section{DSL-007 DOES PHARMACY CONTRIBUTE TO DELAYS IN HOSPITAL DISCHARGE?}

doi:10.1136/ejhpharm-2013-000276.250

V Marvin, S Kuo, D Linnard. Chelsea and Westminster Hospital, Pharmacy Dept, London, UK

Background Efficient management of patient flow including timely discharge from hospitals is vital. Patients in UK hospitals are commonly given individually labelled medicines to take home (TTOs). It is perceived by the multidisciplinary team at our hospital that waiting for these medicines is a significant rate-limiting step in the discharge process.

Purpose We examined the timeframes around TTO prescribing, dispensing and patient discharge in order to identify delays and any negative impact of the pharmacy processes involved.

Materials and Methods All TTO prescriptions entered into the pharmacy electronic log on one day in May 2012 were examined 